\title{
Exposed HIV infants who had their DBS sample collected, tested and received result in past 12 months (January-December 2013) at Specialist Hospital Yola, Nigeria
}

\author{
Article by Jonathan Davou Dung \\ Master of Public Health, Texila American University, Nigeria \\ Email: joedung08@gmail.com
}

\begin{abstract}
This study is a retrospective study design to examine the outcome of babies born to HIVpositive mothers in Specialist Hospital Yola, Adamawa state, Nigeria. The total population of women who tested HIV-positive and accessed antenatal care, intra-natal care and post natal care and were enrolled for PMTCT at Specialist Hospital, Yola between January 2013 and December 2013 and infants below 24 months .

The objective of this study is to determine the number of the exposed infants that received PCR service using DBS as compared with the total number of positive pregnant mothers and also to identify the gaps with the view to correcting them

Total ANC attendee was forty thousand three hundred and Seventy four $(40,374)$ pregnant women of which Eleven thousand three hundred $(11,300)$ received counseled, tested and received results for HIV. Two hundred and Nineteen (219) $1.9 \%$ tested were reported positive. This makes up 1.9\% of the population tested. The HIV testing among HIV-exposed

Infants within the health care facility were (133) $60.7 \%$. The prevalence of HIV infection among

HIV-exposed infants who were tested by HIV-1 DNA PCR method was (8) 8.4\%. 95 (71\%) of the total DBS sample collected had their results. 38 (28.6\%) of the infants never received their result.

The facility had intermittent supply of commodities for EID of HIV, trained human resources, system of identification of HIV-exposed infants. Early HIV testing during

Pregnancy, PMTCT ARV prophylaxis, disclosure of HIV status, enrollment to care and treatment, frequent attendance to EID services, co-trimoxazole prophylaxis and exclusive breast feeding was found to be significant predictors for testing of HIV-exposed infants.
\end{abstract}

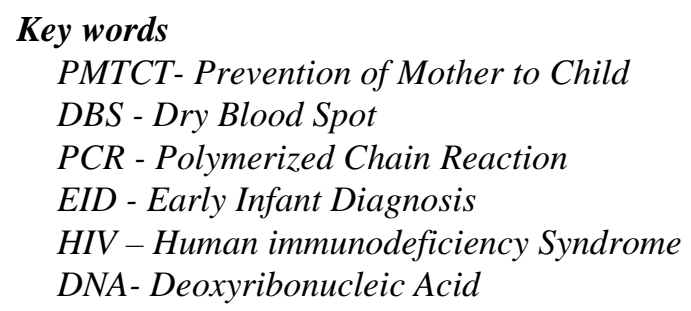

\section{Introduction}

Recognizing the urgent need to intensify the global efforts to eliminate HIV and AIDS to have a society free of AIDS, UNAIDS issued the 2011 Political Declaration on HIV and AIDS to significantly scale up efforts to achieve universal access to integrated HIV prevention, treatment, care, and support programs and services to 15 million people [1]. Despite great efforts and growing access to antiretroviral therapy, morbidity and mortality as relates to HIV remains high in many parts of sub-Saharan Africa. An estimated prevalence of HIV infection in adults between the ages of $15-49$ is $7 \%$ and children less than 5 years is $1.7 \%$ in Uganda [2]. With early case detection of children infected with HIV with the aid of early infant diagnosis (EID) has helped in the early initiation of antiretroviral therapy, thereby 
reducing morbidity and mortality [3, 4]. Currently, EID testing in Nigeria is done by polymerase chain reaction (PCR) analysis of HIV DNA samples in centralized laboratories.

Nigeria in 2006 adopted a simple technique of sample collection and transportation to testing sites using the dried blood spot (DBS) which has made sample collection and transportation from rural setting improve thereby increasing coverage [6]. DBS samples can be transported at room temperature days after initial sample collection, unlike whole blood samples which must be tested on the day of collection [5, 6].

The transport of DBS samples from the lower level health units to referral laboratories for HIV DNA PCR has now been key into the hub and spoke transport network in the state.

The Objective of this study is as follows:

1. To determine the number of the exposed infants that received PCR service using DBS.

2. To identify why the infants could not accessed such services/ To compare the total number of exposed infants that received services with the total number of births

3. To identify the gaps with the view to correcting them.

\subsection{Literature review}

The human immunodeficiency virus (HIV) is the causative agent for the acquired immunodeficiency syndrome (AIDS) ${ }^{[1][2]}$ a condition that the immune system progressively fail thereby allows life-threatening opportunistic infections to thrive. Transmission with HIV infection occurs via breast milk, by the transfer of blood, and seminal and vaginal ejaculates. In these body fluids, the HIV virus are found as free particles as well as virus within infected immune cells.

HIV attacks the human immune system, the $\mathrm{T}$ helper cell predominantly $\mathrm{CD}^{+} \mathrm{T}$ cells, macrophages, and dendritic cells. ${ }^{[3]}$ HIV infection depletes $\mathrm{CD}^{+} \mathrm{T}$ cells to a low level by mechanisms such as apoptosis of cells, ${ }^{[4]}$ direct killing of infected cells, and killing by CD8 cytotoxic lymphocytes. ${ }^{[5]}$ When $\mathrm{CD}^{+}{ }^{+} \mathrm{T}$ cell numbers decline below a critical level, cellmediated immunity is lost, and the body becomes progressively more susceptible to opportunistic infections.

\subsection{Mode of HIV transmission}

HIV virus gets access into the body by way of broken skin, cuts and through the mucous membrane of the vagina and those that are found inside the anus and direct injection by unsterilized sharp objects. There are several ways by which this can happen:

- Sexual contact with an infected person. Anal or vaginal intercourse with an infected partner or a partner with an unknown HIV status without a condom is responsible for the vast percentage of the HIV sexually -transmitted cases.

- Sharing needles, syringes or other injection equipment with someone who is infected. This is commonly found in those who are involved in intravenous drug user (IDU) where they share needles.

- Mother-to-child transmission. The HIV virus is transmitted to the baby before or during birth and through breast milk from a positive mother.

- Transmission in health care settings. Healthcare professionals have been infected with HIV in the workplace, usually after being stuck with needles or sharp objects containing HIV-infected blood.

- Transmission via donated blood or blood clotting factors. However, this is now very rare in countries where blood is screened for HIV antibodies.

People that turns out to be HIV positive may be unaware that they are infected with the virus. $^{[7]}$ in 2001, less than one percent (1\%) of all of the urban population in Africa that are sexually active have tested for HIV, and this proportion is even lower in rural populations. ${ }^{[7]}$ And only $0.5 \%$ of pregnant women attending urban health facilities were counselled, tested or receive their test results. ${ }^{[7]}$ Again, this proportion is even lower in rural health facilities. ${ }^{[7]}$ 
Since donors may therefore be unaware of their infection, donor blood and blood products used in medicine and medical research are routinely screened for HIV. ${ }^{[8]}$

With improvement in modern HIV test, testing is extremely accurate. The predictive value for a single screening test is more than $99 \%$ of the time. ${ }^{[7]}$ The chance of a false-positive result in a standard two-step testing protocol is estimated to be about 1 in 250,000 in a low risk population. ${ }^{[10]}$ Testing post exposure is recommended initially and at six weeks, three months, and six months. ${ }^{[10]}$

A vertically transmitted infection can also be termed as mother to child transmission is an infection that is transmitted to either the embryo, fetus or baby from the mother during pregnancy or childbirth. The causative agent can be bacteria, viruses or, parasites. ${ }^{5]}$

A vertically transmitted infection can be called a perinatal infection if it is transmitted in the perinatal period, which is the period starting at a gestational age of $22^{[5]}$ to $28^{[7]}$ weeks (with regional variations in the definition) and ending 7 completed days after birth. ${ }^{[5]}$

The term congenital infection can be used if the vertically transmitted infection persists after childbirth.

\subsection{Dry blood spot}

Robert Guthrie in 1963 introduced the concept that blood can be obtained from pricking the heel or finger of neonates and blotted onto filter paper using capillary, this technique was used to screen for metabolic diseases in large populations of neonates in Scotland

Since then, the Guthrie card have been to routinely collect samples from infants for varied investigation, such as phenylketonuria , sickle cell disorders , congenital hypothyroidism and HIV infection in over 20 countries of the world

The limitations of sensitivity and specificity when screening such small volumes of blood restricted the use of dried blood spots for many years. However, recent advances such as the production of monoclonal antibodies, expression of synthetic proteins, and the introduction of the polymerase chain reaction have overcome many of these problems. ${ }^{[1]}$

The technology holds promise for expanding diagnostic services to HIV-infected infants in resource-poor settings due to the samples' longer lifespan with reduced need for refrigeration and the less invasive nature of the test compared with other methods. Unlike ELISA testing for HIV-antibodies in the blood, which may be transmitted to infants in pregnancy independently of the virus itself, dried blood spot testing can be used to detect genetic material of the actual virus, thereby avoiding the likelihood of a false positive result. DBS specimens also pose less of a biohazard risk to handlers, and are easier to transport or store than liquid blood specimens. ${ }^{[2]}$

\subsection{Method of sample collection}

The step involved in Sample collection

- Warm the baby's foot or hand depending on the age of the child for $10 \mathrm{~min}$ to facilitate blood flow

- Position the baby with its feet down

- Clean the baby's foot with disinfectant and let it dry.

- While wearing gloves free of powder, the provider pricks the baby's foot with a lancet to draw blood.

- The first drop of blood should be wipe away with gauze or cotton wool

- The provider should allow a large drop of blood to collect on the foot before torching it on the circle on the filter paper

- The foot should then be cleaned with disinfectant and left un-bandaged.

The samples collected should be stored horizontally out of direct sunlight for at-least three hours. Once dried, samples are stored in a sealable plastic bags with desiccant packets and a humidity card and are ready for transport to the Laboratory 
South American Journal of Public Health

Special Edition May 2016

\subsection{Methodology}

This study is a retrospective study design to examine the outcome of babies born to HIVpositive mothers in Specialist Hospital Yola, Adamawa state, Nigeria. The total population of women who tested HIV-positive and accessed antenatal care, intra-natal care and post natal care and were enrolled for PMTCT at Specialist Hospital, Yola between January 2013 and December 2013 and infants below 24 months .This is in accordance with the 2006/2007 WHO guideline of 6 weeks to 18 months of age . A purposive sampling technique was thus adopted for the study.

\subsection{Result}

The following records were consulted during the study:- these records are

1. ANC attendant register

2. PMTCT register

3. Child follow -up register and the

4. Dry Blood Spot register.

A total of forty thousand three hundred and Seventy four $(40,374)$ women attended the Anti-natal clinic, out of which Eleven thousand three hundred $(11,300)$ were counseled, tested and received results for HIV.

Two hundred and Nineteen (219) of the Eleven thousand three hundred tested, were reported to be positive. This makes up $1.9 \%$ of the population tested. The HIV testing among HIV-exposed

Infants within the health care facility were (133) 60.7\%. The prevalence of HIV infection among

HIV-exposed infants who were tested by HIV-1 DNA PCR method was (8) 8.4\%. 95 (71\%) of the total DBS sample collected had their results. 38 (28.6\%) of the infants never received their result.

The facility had intermittent supply of commodities for EID of HIV, trained human resources, system of identification of HIV-exposed infants. Early HIV testing during

Pregnancy, PMTCT ARV prophylaxis, disclosure of HIV status, enrollment to care and treatment, frequent attendance to EID services, co-trimoxazole prophylaxis and exclusive breast

Feeding was found to be significant predictors for testing of HIV-exposed infants.

\section{Charts}

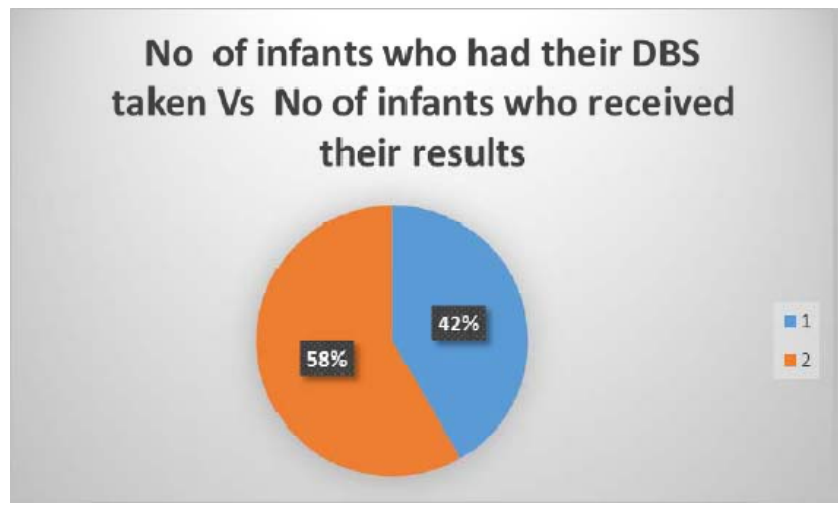




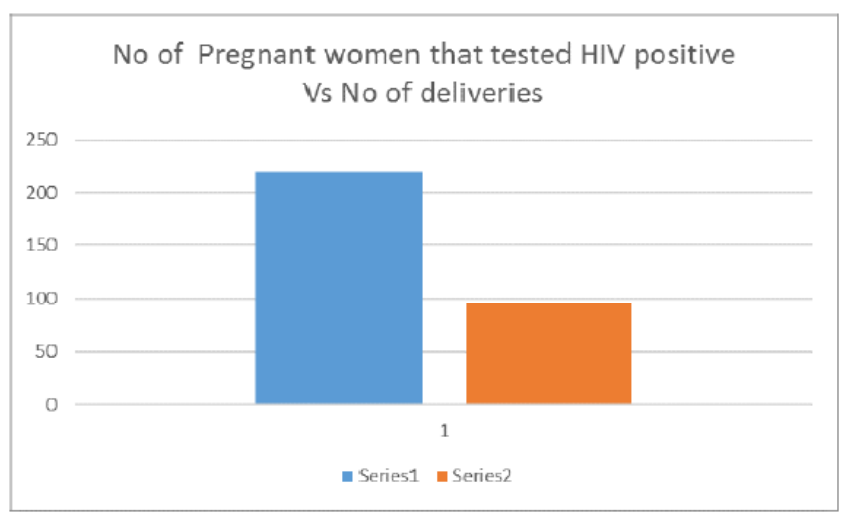

\subsection{Discussion}

Early infant diagnosis (EID) of HIV infection provides the opportunity for identifying, testing and subsequent follow up of HIV-exposed infants. This process benefits both the HIVinfected infants, the uninfected infants with their family members once they are properly counseled, linked to holistic HIV care and support, monitoring growth and child development and safe infant feeding options. In Nigeria, despite of availability of EID of HIV infection testing services, many children are left undiagnosed or diagnosed late that resulted to increased childhood HIV related mortalities

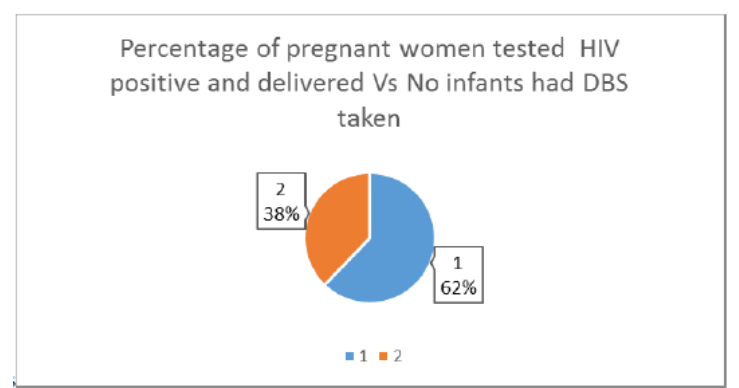

\subsection{Proportion of HIV testing in infants and young children}

A total of $67.5 \%$ of the infants that visit the facility have accessed to be tested. This when compared with other studies done elsewhere, it agrees with 67\% reported in Kenya (8). The study findings do not agree with what was reported previously in Tanzania (10) which was $87 \%$.

However, 38 (28.6\%) who had access to HIV EID services but were unable to receive their results More efforts are needed to maximize identification and testing of HIV-exposed infants by linking EID of HIV infection testing with other potential entry points like outpatient clinics, pediatrics ward and immunization clinics. The current national algorithm for EID of HIV infection recommends testing of HIV exposed infants at the age of 4-6 weeks or any time thereafter (WHO EID guideline). The increase in uptake of EID testing, can further be contributed by more sensitization through client counseling, health talk in health facility, increased awareness on EID of HIV infection and availability of commodities for DBS collection.

\subsection{Prevalence of HIV infection among HIV exposed infants}

The finding that overall 8(8.4\%) of HIV-exposed infants tested positive using HIV-1 DNA PCR test on their first test is comparable with $17 \%$ documented previous in Tanzania (10) and $16 \%$ in Mozambique (Cook et al 2011) Rapid scale up of PMTCT services, wide coverage of PMTCT intervention with phased out of less efficacious single dose NVP, introduction of more efficacious PMTCT regimen and ART could have contributed to the decrease in HIV positivity rate among HIV-exposed infants. 
South American Journal of Public Health

Special Edition May 2016

However, more effort should be considered by endorsing global approaches towards elimination of mother to child transmission of HIV as targeted to be below 5\% (6). The strategies involve maximizing PMTCT coverage to remote areas, availability of commodities for HIV testing in mothers and children, enhancement of skills for health care providers and effective initial and continuous utilization of HIV services.

Furthermore, prioritization of maternal ART reduces risk of HIV transmission to exposed infants.

\subsection{Conclusion}

The state, and by extension the Federal government should focus on implementation of global plans for elimination of Mother to child HIV transmission (e-MTCT) this can be achieve in collaborating with stakeholders by involving them in the design of cost effective approaches that will lead to strengthening the system at all levels of healthcare facilities thereby Suring up of EID services.

The various level of health administrators should ensure that the supply for PMTCT and EID commodities for proper service delivery is not interrupted.

Awareness should be created on the importance of HIV testing, prevention of mother to child transmission of HIV during pregnancy and proper infant/mother follow up HIV intervention among women of childbearing age in the community.

Availability of efficacious drugs, commodities for testing, prevention from mother to child transmission services and skilled health care manpower has led to high uptake of EID services thus the decreased prevalence of HIV infection.

\subsection{Limitation}

This study was only conducted in one facility out of the many that provide similar services. With this at the back of our mines, the outcome of this study might not give a true representation of the EID services that is being provided across the length and breadth of Nigeria in general and Adamawa state in Particular.

The study was centered on reviewing records and the actions were not involved to find out their views as to why certain things were not done.

\subsection{References}

[1.] (n.d.). Retrieved April 7, 2014, from www.poz.com/arcchives/2008_march_2168

[2.] Aledort JE, R. A. (2006). Reducing the burden of HIV/AIDS in Infants: the contribution of improved diagnostics. Nature, 444 suppl 1. 19-21.

[3.] Assembly, U. G. (2011). Political declaration on HIV and AIDS: Intensifying our efforts to eliminate HIV and AIDS. Geneva: UNAIDS.

[4.] Cassol S, S. T. (December 1992). Stability of dried blood spot specimens for detection of human immunodeficiency virus DNA by polymerase chain reaction. J. Clin. Microbiol.30 (12), 3039-42.

[5.] Chou R, H. L. (2005). Screeening for HIV: a review of evidence for the U.S Preventive Service Task Force. Annals of Internal Medicine143(1), 55-73.

[6.] Ciaranello AL, P. J.-A. (2011). Early infant HIV-1 diagnosis programs in resource-limited settings: opportunities. BMC Med.

[7.] Douek DC, R. M. (2009). Emerging Concepts in the Immunopathogenesis. Annu. Rev.Med 60, 471-84.

[8.] en wikipedia.org/wiki/infection

[9.] en wikipedia.org/wiki/Dried_Blood_Spot

[10.] Generalized epidemic in sub Saharan Africa. Journal of international development 13(4) 451-466

[11.] HIVand AIDS. Geneva: UNAIDS

[12.] Hassan AS, S. E. (2012). .Dynamics and constraints of early. AIDS and Behavior. 16(1), 5-12.

[13.] Kumaranayake L, W. C. (2001). Resource allocation and priority setting of HIV/AIDS interventions: addressing the generalized epidemic in sub-saharan Africa. Journal of international Development 13(4), 451-466. 
[14.] Muhimbili University of Health and Allied Science by JG Gamaliel 2012

[15.] Nuwagaba H.B., S. W. (2010). Introducing a multi-site program for early diagnosis of HIV. BMC Pediatr.

[16.] Parker SP, C. W. (September 1999). The use of the dried blood spot sample in epidemiological studies. J. clin. pathol.52 (9), 633-9.

[17.] Post exposure prophylaxis against human immunodeficiency virus. American Family Physician 82(2):161-6

[18.] RA, W. (1993). How does HIV cause AIDS ? Science 260(5112), 1273-9.

[19.] Stability of Dry Blood Spot specimen for detection of human immunodeficiency virus DNA by polymerase chain reaction. J.clin.microbiol.30 (12):3039-42

[20.] Schools-wikipaedia.org/wp/h/hiv.htm

[21.] S, K. (2004). Patient inforation: Blood donation and transfusion. uptodate.

[22.] Tolle MA, S. H. (July 15, 2010). Post exposure prophylaxis against human immunodeficiency virus. American Family Physician 82 (2), 161-6.

[23.] Wikipedia Dry Blood Spot, 3/27/14

[24.] www.Pathfinder's.org , early infant diagnosis

[25.] www.emtct-1qtt.org : utilization of HIV and AIDS -mother to child transmission

[26.] www.Independent.org/ Delusions of power by Robert Higgs

[27.] www.update.com

[28.] www.plosone.org

[29.] www.scirp.org/journal/paper download. J. Purushottam, 2012

[30.] www.youtube.com question/index?qid

[31.] www.intergayhiv.com/Humanimmunodeficiencyvirus-hiv

[32.] www.Ihaveachronicdisease.com/hivaids

[33.] www.POZ.COM/archives/2008_mar_2168.shtml

[34.] www.andrenaingram.org/always-to-contract-hiv

[35.] www.palneetclinic.com/index

[36.] www.sanfordburnham.org 